\title{
DIE SLAVIA SUBMERSA IN ÖSTERREICH: EIN ÜBERBLICK UND VERSUCH EINER NEUBEWERTUNG
}

\section{Allgemeines}

Was das slawische Lehngut Österreichs und insbesondere Kärntens betrifft, ist festzuhalten, dass es sich auf Grund linguistischer Fakten als größtenteils sehr alt erweist, obwohl das Meiste davon erst relativ spät überliefert wird. Doch einige slawische bzw. slowenische Toponyme sind urkundlich schon vor dem Jahre 1000 belegt (u.a. in Kärnten 6), zwischen 1000 und 1250 nimmt die Belegdichte zu. Bei den Personennamen werden vor 1000150 „Slawen“ genannt, nach 1000 über 380. ${ }^{1}$ Sie scheinen alle in lateinisch oder deutsch geschriebenen Urkunden auf. E. Kranzmayer hat eine Reihe von Anhaltspunkten für die Chronologie der Übernahme von Ortsnamen ins Deutsche geliefert ${ }^{2}$. In einem größeren Zusammenhang entspricht die slawische Sprachform, die den Ortsnamen im Osten und Süden Österreichs (Osttirol, Kärnten Steiermark, Salzburg-Lungau, südliches Nieder- ${ }^{3}$ und östliches Oberösterreich) zu Grunde liegt, dem „Alpenslawischen“ (nach Ramovš ${ }^{4}$, Kronsteiner ${ }^{5}$ usw.) bzw. der Sprache der altslowenischen "Freisinger Denkmäler"6, was auch bereits der große slowenische Dialektologe und Sprachhistoriker Ramovš festgestellt hat. Die Varianten in der deutschen Wiedergabe der slawischen Namen wollte er dialektologisch deuten, mir ist es aber gelungen, diese Unterschiede chronologisch zu erklären, woraus folgt, dass es im hohen und späten Mittelalter eine über Kärnten hinausgehende weit verbreitete Gemischtsprachigkeit in Österreich $\mathrm{gab}^{7}$, was sich auch in alten Lehnwörtern widerspiegelt. ${ }^{8}$

Diese Gemischtsprachigkeit des frühmittelalterlichen Slawentums in Österreich stellt Holzer in einem sehr inhaltsreichen Beitrag recht anschaulich dar ${ }^{9}$ (s. 4.0). Diese Sprache weist einige Besonderheiten auf, die sich in den slowenischen Kerngebieten nicht finden, u.a. den Ortsnamentyp auf -iče (s. 3.3) und das häufige Wortbildungselemt -nik (eingedeutscht meist -nig, s. 3.2) sowie einige Wörter ${ }^{10}$ (s. 4). Es erhebt sich nur die Frage, wie man diese Sprache nennen soll, „slowenisch“ im heuti-

\footnotetext{
1 nach Kronsteiner 1975b: 110ff.

2 Kranzmayer 1956: Kap. V u. VI.; dazu vgl. auch Pohl 2000: 37ff. mit Lit.

3 dazu vgl. Holzer 2001.

4 v.a. Ramovš 1936: $23 \mathrm{ff}$.

5 v.a. Kronsteiner 1975b: $140 \mathrm{ff}$.

${ }^{6}$ dazu vgl. zuletzt Pohl 2002a: 177ff. (mit Lit.) sowie 2002b: 53ff. (mit Lit.).

7 worauf in letzter Zeit auch Holzer in zahlreichen Arbeiten hingewiesen hat (s. Holzer 2002a-c)

8 zum deutsch-slawischen Sprachkontakt in Österreich s. Pohl 1997c u. Neweklowsky 1997.

${ }^{9}$ Holzer 2002a.

10 dazu schon früher Pohl 2002a: v.a. 179 bzw. 2002b: 68f.
} 
gen Sinn ist sie nicht, "alpenslawisch" ist zu allgemein, daher denke ich, der beste Name wäre Karantanisch, da die Slavia submersa der österreichischen Alpenländer die Sprache des Karantanen-Reiches war ${ }^{11}$, der ersten historischen Staatsgründung der Vorfahren der heutigen Slowenen. In den Abschnitten 2-4 soll zunächst das Material präsentiert und in 5 dessen Beurteilung vorgenommen werden.

\section{Phonologisches}

Für das relativ hohe Alter des Sprachgutes slawischer bzw. slowenischer Herkunft in Österreich spricht, dass sich bereits in den (altslowenischen) Freisinger Denkmälern Sprachformen finden, wie wir sie auch in der alpenslawischen Onomastik beobachten können, und zwar ${ }^{12}$ :

1. Erhalt der Nasalvokale (Freising vuensih 'vęštichъ / größer [GLpl.]', malomogoncka 'malomogọsta / krank, schwach', sunt 'sọtb / sind' usw.) ${ }^{13}$ (s. 2.1);

2. urslaw. ' $t j>t{ }^{\prime} \sim k^{\prime}$ ' (Freising choku 'choštọ / will', imoki 'im@šti / habend [Ipl.]' usw. $)^{14}$ (s. 2.2);

3. urslaw. ${ }^{*} s t j / s k j / s k^{e, i}>\check{s c}$ (Freising crisken / cristen 'krišteno / getauft', este 'ešte / noch' usw. $)^{15}$ (s. 2.3);

4. Erhalt der Lautgruppe $d l$ (Freising modliti (ze) 'moliti (sę) / beten' usw.) ${ }^{16}$ (s. 2.4);

5. Fehlen des prothetischen $j$ - (Freising este [s.o.], ego-/emu-se 'jego-/jemu-že / seiner / ihm' usw.) ${ }^{17}$ (s. 2.5).

Darüber hinaus gibt es Lautungen, die in die vorliterarische Frühzeit des alpinen Slawisch weisen, wie z.B. das Unterbleiben der Liquidametathese (in dt. Talggen, s. 4.15) oder $\breve{a}$ statt $o$ (in dt. Kraxe, s. 4.12, und eben auch in Talggen).

2.1. Erhalt der Nasalvokale: Der Nasal $e$ ist auffallend selten belegbar (z.B. BN Lending, Granatspitzgruppe, Osttirol < *lędina oder -nikъ 'Brachland', SN Fentsch,

11 Zur Begrenzung s. Poh1 2002a: 184f. (mit Lit.) u. 2002b: 73, vgl. auch Holzer 2001: 50 sowie hier 4.18 samt Anmerkungen.

12 Nur anhand dieser hier diskutierten 6 Punkte lassen sich eindeutige Beziehungen zwischen der Sprache der Freisinger Denkmäler und den österreichischen ON herstellen. Vgl. weiters: Ramovš 1936: 22, Pohl 1996a, Cvetko-Orešnik 1989: 35ff., insb. 50, zuletzt 1996.

13 Die Entstehung der Nasalvokale erfolgte seit dem 7. Jhdt. (Shevelov 1964: 633 u. 311ff.), vgl. auch Ramovš 1936: $26 \mathrm{ff}$.

${ }^{14}$ Sichere Fälle von urslaw. ${ }^{*} d j>j$ kommen außerhalb des gemischtsprachigen Gebiet Kärntens nicht vor (auch in den alpenslaw. PN nicht, vgl. Kronsteiner 1975b: 137). Ein Kandidat für slaw. ${ }^{\star} d j$ ist der Name Colomezza (NÖ) < *kolo-medja 'Pfahl-grenze' mit westslaw. Entwicklung zu /kolomeza/ (Holzer 1991: 51f., zuletzt Bergermayer 2005: 108f.). Die in ANB I 203 erwogene Kärntner Entsprechung dieses Namens in Kärnten (Colmez) ist unsicher, m.E. v.a. aus lautlichen Gründen, da in Kärnten *dj > $j$ wird (der betreffende Name geht wohl eher auf *chblmbcb 'Berg, Hügel' zurück). - Für Shevelov (1964: 212) gilt nur * $t j>$ slow. $\check{c}$ - ohne auf das alpenslaw. Namenmaterial näher einzugehen. Vgl. auch Ramovš 1936: 50ff.

15 Shevelov (1964: 213) stellt lapidar fest, dass das Ergebnis slow. $\breve{s c ~ s e i ~(s . o . ~ A n m . ~ 14) . ~-~ R a m o v ̌ s ~(1936: ~ 53) ~}$ glaubt einen Unterschied zwischen den altslowenischen Repräsentanten von ${ }^{*} s k j /{ }^{*} s k^{e, i}$ und ${ }^{*} s t j$ erblicken $\mathrm{zu}$ können, doch das von ihm zitierte crisken wird auch cristen geschrieben.

16 Die Vereinfachung der $t / / d l$-Gruppen $>l$ erfolgte im 8./9. Jhdt. (Shevelov 1964: 634 u. 370ff.). Der Erhalt dieser Gruppen ist für die slaw. Dialekte von den Alpen bis zu den Karpaten (und nördlich davon) charakteristisch (Shevelov 1964: 373). Vgl. auch Ramovš 1936: 47ff.

17 Die $j$-Prothese bei $e$ - setzt mit dem 6. Jhdt., die bei $a$-im 8./9. Jhdt. ein (Shevelov 1964: 633 u. 634). 
Gem. St. Marein bei Knittelfeld, St < Vętj-, zu ${ }^{*} V e ̨ t j e s l a v b$ wie u.a. altčech. Váceslav 'Wenzel', russ. Vjačeslav usw. 'größeren Ruhm habend'), o ist sehr häufig, z.B. slaw. *loka 'feuchte Wiese' in Landschütz (FN, Gem. Matrei i.O.), Lonza (BN, Gem. Mallnitz), Lang (2x, SN, Gem. Ferndorf und Feldkirchen), Langen (SN, Gem. St. Andrä), Lansach (SN, Gem. Weißenstein), Laing (SN, Gem. Spielberg bei Knittelfeld), Lang (SN, Gem., Leibnitz). In jüngeren slow. (also relativ spät ins Deutsche gelangten) Ortsnamen ist kein Nasalvokal mehr vorhanden, z.B. Laak / Loka (SN, Gem. Ferlach). Der Erhalt der Nasalvokale widerspiegelt das Alter des Namengutes. Je früher ein Name ins Deutsche gelangt ist, desto eher erscheint ein Reflex des Nasalvokales, übrigens auch in PN, z.B. Zuentipolch 'Svętipblkъ / Svatopluk usw.', Zanto / Zunducu 'Sodъ(kb)' zu sqdb 'Richter' (wohl Kurzform zu einem zusammengesetzten PN) ${ }^{18}$. Ein besonderes Charakteristikum der Slavia submersa ist darin aber nicht zu erblicken $^{19}$, doch es ist ein Zeugnis hoher Altertümlichkeit, die auch alte Lehnwörter wie Munggen (4.15) oder Strankerl (4.21) sowie Pränter (4.19) zeigen.

2.2. urslaw. ${ }^{\star} \mathbf{t j}(b z w . \mathbf{k t i})>\mathbf{t}$ ' $\mathbf{k}$ ': In Randlagen sind nur solche Lautungen greifbar, wie u.a. Defereggen in Osttirol $\left(<{ }^{*}\right.$ Dobrik'e zu einem mit dobro 'gut' anlautenden PN, vgl. den alpenslaw. PN Thabricho, 8. Jhdt., d.i. ${ }^{\star}$ Dobritjb $\left./ k^{\prime} b\right)^{20}$ oder Flattach in Oberkärnten (Gem., zu *blatje [Lpl.], Einwohnername zu blato 'Sumpf'), ebenso in früh deutsch gewordenen Gebieten (wie in der Steiermark, z.B. Maßweg, Gem. Spielberg bei Knittelfeld < *Měšovik'e, 1295 Messbich), aber auch bei früher Entlehnung, z.B. in Kärnten Radweg / Radoviče (Gem. Feldkirchen < ${ }^{*}$ Radovik'e) oder Pöckau / Peče (Gem. Arnoldstein) bzw. Peggau (St, ca. 1050 Pecah, 1138 Bekach, < 'Pek'e, Einwohnername zu slow. peč 'Fels, Ofen' < urslaw. *pekti-bzw. * ${ }^{*}$ etj-). Auch hier ist das Ortsnamengut beredter Zeuge des altslowenischen Zustandes. - Vgl. auch 3.3.

2.3. urslaw. *stj / skj / sk ${ }^{\mathrm{e}, \mathrm{i}}>$ altslow. šć > dt. šk / št (heute schk / sk / st) bzw. slow. šč: Diese Lautung ist v.a. im Wortbildungselement (nomina loci) slow. -išče besonders häufig. Wenn man das Material betrachtet, entsteht der Eindruck, Oberkärnten und Osttirol sowie Salzburg (v.a. Lungau) und Teile der Steiermark hätten $s(c h) k$, aber Unterkärnten und der größere Teil der Steiermark st - Reflex alpenslawischer dialektologischer Verhältnisse ${ }^{21}$. Doch vielmehr scheint es so gewesen zu sein, dass ursprünglich überall das altslow. šć sehr palatal ausgesprochen wurde und in früh eingedeutschten Gebieten als schk übernommen wurde wie z.B. Staniska (SN, Kals $<$ *stanišće 'Standort, Lagerplatz') in Osttirol, Malesischk (FN, Gössnitz bei Heiligenblut < *molzišč 'Ort, wo man melkt; Melkerei, Melkstätte') in Kärnten oder Patschgen (SN/FN, Gastein < *pašišce 'Weideplatz') in Salzburg. Dann ist diese Lautgruppe in Richtung neuslow. šc hin entwickelt worden, was dann im Deutschen mit $s t$ wiedergegeben wurde bzw. im grössten Teil des Bairischen st ergab, daher haben wir z.B. Gassarest (SN, Gem. Strassburg < *kozarišće 'Ort der Ziegenhirten') in

\footnotetext{
18 Kronsteiner 1975b: 65.

19 Zumal in den Jauntaler Mundarten bis heute die Nasalvokale z.T. erhalten sind (vgl. Zdove 1972: 74ff).

${ }^{20} \mathrm{Zu}$ diesem Namen vgl. Pohl 1996b: 42 u. 60 mit Lit. und anderen Erklärungsvorschlägen. Vgl. überdies in Kärnten den SN Dobritsch gleicher Herkunft (Gem. Friesach u. Guttaring, 930 mons Doborich).

21 Dies habe ich voreilig in Pohl 1996a: 312f. behauptet, Ramovs (1936: 53) folgend.
} 
Unterkärnten oder Liesing (GN/SN < *lěśćcnica zu lěsbkb 'Haselnuss', 9./10. Jhdt. Liestinicha) in der Steiermark. In den meisten Kärntner slowenischen Mundarten ist $\check{s} \check{c}>\breve{s}$ geworden wie z.B. Homölisch / H(o)mel(i)šse (SN, Gem. St. Margareten im Rosental und Zell < *chrmèljišćc zu slow. hmelj 'Hopfen'), eine Lautung, die auch der Steiermark nicht fremd ist, z.B. Fladischerhof (HN, Gem Turnau, Bruck a.d. Mur < *blatišce zu slow. blato 'Sumpf').

2.4. d1-Gruppe: Der Erhalt dieser Gruppe gilt als Charakteristikum des Westslawischen. Da das Alpenslawische ursprünglich die Brücke zwischen dem West- und Südslawischen bildete, ist es nicht verwunderlich, dass sich auf diesem Areal beide Ergebnisse zeigen. Noch heute zeichnet sich der Gailtaler slowenische Dialekt durch das Bewahren von $d l$ aus. Sprachgeographisch ist in dieser Hinsicht das Alpenslawische ein Übergangsgebiet, auf dem überwiegend südslawische Merkmale mit westslawischen Zusammentreffen 22 .

2.4.1. dl bewahrt: (Osttirol) Tscharniedling (SN, Gem. Dölsach, zu *črrnidlo 'schwarzer Ort', slow. črn 'schwarz'), Welzelach (SN, Gem. Virgen, 1300 Welcedlach, Lpl. zu *velese(d)lje 'Einwohner des großen Dorfes'); (K) Matschiedl / Močidlo (SN, Gem. St. Stefan a.d. Gail) u. Hart / Vočilo bzw. Ločilo (SN, Gem. Arnoldstein, ma. Voči(d)lo, beides 'feuchter Ort', dt. Hart 'Sumpfwald'), Pojedl (SN, Gem. Himmelberg, 1431 Pogedl < slow. *po je(d)li 'bei der Tanne' oder *pojedlbje 'Tannengegend'); (St) Elz (SN, Gem. Puch bei Weiz, 1318 Edlncz < slow. *(j)edlbnica 'Tannengegend'), Irdning (GN/SN, Gem., 1185 Jedenich [GN], 1140 Idinich [SN] < slow. "jedlbnika 'Tannenbach'), Muntschiedel (1495, abgekommener SN bei Frohnleiten, wie Matschiedl, s.o.); (Salzburg) Jedl (SN, Gem. Muhr, < slow. je(d)la 'Tanne'); (NÖ) Mödelsdorf (SN, Gem. Emmersdorf a.d. Donau, 1184 Modelansdorf, zum slaw. PN Modlena o.ä.), Modlisch (SN, Gem. Schwarzenau, Allentsteig, 1150 Modlisse, zum slaw. PN Modlišb o.̈.), Modsiedl (SN, Gem. Raabs a.d. Thaya, 1242 Motsidel, wie Matschiedl, s.o.), Modzidala (1067, abgekommener SN bei Marchegg, wie vor.), Edlitz (SN, Gem. Aspang, 1192 Edelz, zu slaw. je(d)la 'Tanne'), Ödlitz (SN, Gem. Berndorf, 1395 Edliczen, wie vor.), Edlitz (SN, Gem. Weinburg, 1072/91 Hediliz).

2.4.2. d1 > 1 (bzw. Erhalt von d1 nicht nachweisbar): (K) Dulieb (1060-70, abgekommener SN bei Spittal a.d. Drau, zum slaw. Stammesnamen Dudleben), Mötschlach (SN, Gem. Irschen, 1300 Mötzlach zu slaw. *moči(d)lo 'feuchter Ort'), (St) Mötschlach (SN, Gem. Oberaich, Bruck a.d. Mur, 1293 Moetslach zu slaw. ${ }^{*}$ moči(d)lo 'feuchter Ort'), (NÖ) Metzling (SN, Gem. Persenbeug, 1197 Moczelicz < slaw. *močidlbce o.ä. 'feuchter Ort').

Zum Problem se(d)lo 'Dorf' vs. sedzlo 'Sattel' (s.a. 3.4): Diese beiden Appellativa sind nur schwer voneinander zu trennen ${ }^{23}$. Weder sind alle in Frage kommenden Ortsnamen als 'Sattel' aufzufassen noch dürfte 'Dorf' besonders häufig sein. Mit einiger Wahrscheinlichkeit gehen also folgende Namen auf sedblo 'Sattel' zurück:

22 Siehe Karte 7 bei Mader 1986 (allerdings ungenau) für die Steiermark bzw. Karte 3 bei Pohl 1996a für Kärnten und Osttirol (mit dem Fehler, Zeltschach [Nr. 102f.] nicht als 'Sattel' verzeichnet zu haben).

23 Ramovš 1936: 47 zieht nur (wie er es schreibt) sedlo 'Sitz, Besitz' in Betracht; in einem ähnlichen Sinne auch Kronsteiner 1975a. Kranzmayer (1958: 255f.) wiederum will nur 'Sattel' gelten lassen. 
(Osttirol) Zedlach (SN, Gem. Matrei i.O., 1022-39 Cetulic < slaw. *sedblik'e [Einwohnername] 'die am Sattel wohnen'), Zettalunitzkees (FN, Venedigergruppe, kein urk. Beleg < slaw. *sedblovbnica 'Sattelkees, der vom Sattel herunterkommende Gletscher'); (K) Zedlitzdorf (SN, Gem. Gnesau, 1281 aput Zedeltz, slow.ma. Sedlice), Zedlitzberg (SN, Gem. Himmelberg, 1253 Zedelz, 1267-68 Nazzedele, slow.ma. Na sedle), Zeltschach (2x, SN, Gem. Gurk u. Friesach, 1060-88 Zedelzach [Gurk, benachbart SN Sattelbogen]), Zedelnig / Sedelnik (HN, 'Sattelbauer', Gem. Techelsberg); (St) Zitoll (SN, Gem. Deutschfeistritz, 1383 Cytol), Selzthal (SN, Gem., 1080 Edilts(c)ach, 1100 Cedelse), Zettelbauer (HN, Krieglach, 1280-95 Cedel, 1416 im Zedel). Ferner (wegen der Lage) Zedl und Zödl (SN, bei Radenthein) und Hohe Zölz (BN, Vordernberg, ca. 1300 Zelczaw).

Kandidaten für se(d)lo 'Dorf' sind zunächst die Ortsnamen des gemischtsprachigen Gebietes von Kärnten wie Zell / Selo (Gem. Ma. Saal), Zell / Sele (< Einwohnernamen Selje, Gem., südlich Ferlach), Obersielach / Sele (Gem. Völkermarkt), Sala / Selo (Gem. Feistritz im Rosental), Seel / Selo (Gem. St. Margareten im Rosental), Seelach / Selo (Gem. Sittersdorf), Vesielach / Vesele ( $<$ novoselje 'Neusiedler [Einwohnername]'). Sie sind ins Deutsche erst entlehnt worden, als der Wandel $d l>l$ im Slowenischen schon abgeschlossen war. Dazu kommen nun diejenigen Toponyme, die relativ früh ins Deutsche entlehnt worden sind und deren Lage die Annahme eines 'Sattels' unwahrscheinlich erscheinen lässt: (Osttirol) Welzelach (s.o. 2.4.1), Zöttl (HN, Kals, stimmt semantisch mit *Fig 'Dorf' überein $\left.{ }^{24}\right)$; (K) Zedl (2x, SN, Gem. Frauenstein, 1570 Potzedl und Gem. Kappel am Krappfeld, 1296 Zedlach), Zödl (SN, Gem. Steinfeld); Zerlach (SN, Gem., Feldbach, 1265 Cedlach, Zedlach); (NÖ) Ober-/Unter-Edlitz (SN, Gem. Thaya, 1136 de Cedelz, 1230/1400 Edlicz); (Oberösterreich) Edlhof (HN, Gem. Windhag bei Perg, 1668 Zetlhof).

2.5. Fehlen des prothetischen j:- Sprachgeographisch ist die Entwicklung des prothetischen $j$-vom Zentrum des slawischen Sprachgebietes ausgegangen und an den Rändern entweder nicht konsequent oder überhaupt nicht durchgeführt. Solche Randlagen sind Oberkärnten und Osttirol (letzteres durchwegs ohne $j$-), z.B. Arnig (SN, Gem. Kals) und Auernig (BN, Gem. Mallnitz), beide < slaw. *avorbnikz zu slow. javor 'Ahorn' gegenüber gleichbedeutenden Jauernigg (HN, südlich Predlitz, St) oder Jauring (SN/GN, Gem. Aflenz). Das benachbarte Aflenz (< *ablan(bn)ica, 1025 Auelniz) weist kein $j$-auf, was eindeutig in der Chronologie begründet ist ${ }^{25}$, wie u.a.. auch dt. Aßling gegenüber Jesenice (zu *asenb bzw. slow. jesen 'Esche') in Slowenien. Es liegen also chronologisch bedingte gemischte Verhältnisse vor.

\section{Morphologisches}

3.1. bratro 'Bruder' (Freising bratra, bratria): Die beiden SN Fratres (K, Gem. Spittal a.d. Drau u. NÖ, Gem. Waldkirchen a.d. Thaya ${ }^{26}$ ) sind von einem auf slaw. *bratrı beruhenden PN abgeleitet, etwa *Bratrěšb. Ein dritter Beleg liegt im SN Drat-

24 vgl. Odwarka-Pohl 1998: 125.

25 Vgl. auch die Karte 2 bei Mader 1986.

26 Dieses zweite Fratres (NÖ) kann auch ein dort recht häufiger genitivischer SN sein (vgl. Bergermayer 2005: 40). 
trum vor (Gem. Klein St. Paul, K, urk. 1188-93 Fratran, 12./13. Jhdt. Vratran), das auf ein altes slaw. *Bratrańb zurückgeht. ${ }^{27}$ Die Form bratr- gilt als "westslawisch", wie auch der Erhalt der $d l$-Gruppe (s. 2.4), ist aber auch in den Freisinger Denkmälern bezeugt und somit altslowenisch bzw. karantanisch.

3.2. -nik: Ein besonderes Charakteristikum der Slavia submersa Süd- und Südostösterreichs bzw. des Alpenslawischen sind die zahlreichen Hof- und Familiennamen (urspr. Lagenamen) auf -nig(g) (auch -nich, -nick, nigkh, -nik und -nikch) aus slow. -nik, die in den dem zusammenhängenden slowenischen Sprachraum vorgelagerten deutschsprachigen Gebieten Kärntens, Osttirols, des Salzburger Lungaus und der Steiermark weitaus häufiger sind als im slowenischen Kerngebiet; sie können als „nordslowenisch“ bezeichnet werden. ${ }^{28}$ Es ist auch heute noch produktiv, in der slow. Toponymie begegnet es vor allem in Haus- und (häufig davon abgeleiteten) Familiennamen sowie in Oronymen. ${ }^{29}$

Wie Hornung gezeigt hat, greift das slow. -nik-Suffix in der bair-slaw. Kontaktzone Kärntens und Osttirols auch auf dt. Sprachmaterial über, wodurch es zu dt.slaw. Mischbildungen im Bereich der Hofnamen kommt. Einige von ihr in diesem Zusammenhang genannten Beispiele sind Eggenig (zu dt. Egge) und Waldnig (zu dt. Wald). Hornung kommt zu dem Schluss, dass dt. -er und slaw. -nig bis zu einem gewissen Grad austauschbar waren. ${ }^{30}$ Diese These kann mit zwei Beispielen aus Osttirol erhärtet werden. Eindeutig dt. Etyma weisen die Hofnamen Albernig ${ }^{31}$ und Watschgernig 32 auf. Bei beiden Namen tritt in den ältesten Urkundenbelegen aus 1385 die Endung -nig noch nicht auf, diese erscheint zum ersten Mal im jeweils zweitältesten Beleg aus 1545, bleibt dann jedoch in den schriftlichen Dokumenten durchgehend bis ins 19. Jh. am Namen haften. In einem hinsichtlich seiner Sprache auffallend dialektnah gehaltenen Verzeichnis aus 1775 scheint Watschgernig als Watschernig, daneben jedoch auch als Watscher und Watschgers (G) auf, woraus geschlossen werden kann, dass die Endung damals offenbar bereits abgestoßen war. Im selben Dokument steht für Albernig sowohl Albernig als auch Alber. Nicht auszuschließen ist freilich auch, dass es sich bei der Form mit -nig um eine vom tatsächlichen Sprachgebrauch unabhängige Kanzleiform handelt. ${ }^{33}$ Auf die erwähnte

27 später umgeformt (vgl. ANB I 273, Kranzmayer 1958: 55).

28 Ähnlich Kranzmayer 1956: 180. Er sieht darin nicht zuletzt einen gewissen Einfluss des Dt., die -nik-Namen seien nach dt. Muster gebildet worden, wobei das slow. Suffix dabei dem dt. er entspricht. Bei den meisten dieser Namen auf-nik handelt es sich ihm zufolge um Lage- und Beschaffenheitsnamen, welche meist über ein dt. Pendant verfügen. - Zur Herkunft des Suffixes s. jetzt Bergmann 2002: 335.

29 vgl. die Zusammenstellungen von dieses Suffix enthaltenden Osttiroler Namen bei Bergmann 2002: 335f. und Pohl 1996: 55, für Kärnten bei Kranzmayer 1958: 164. - Im Slawischen des Erlaftales begegnen ebenfalls Namen auf slaw. -nikb, allerdings keine Haus- bzw. Hofnamen (vgl. das Material bei Holzer 1991, insbes. 123).

${ }^{30}$ Hornung 1981: 66. Diese Austauschbarkeit wurde auch in Kleinkirchheim in Kärnten beobachtet (vgl. Bergmann a.a.O.).

31 Alber bezeichnet in weiten Teilen des bair. Gebietes, darunter auch in Tirol und Kärnten, unterschiedliche Arten der Pappel (populus), vereinzelt auch der Ulme (ulmus) und Weide (salix). Vgl. WBMÖ I 132.

32 Als Etymon dieses Namens ist mhd. watschar anzusehen, welches 'abgabenpflichtiges Gut' bzw. 'Abgabe eines solchen' bedeutete (lt. Bergmann a.a.O.).

33 so Bergmann a.a.O. 
Austauschbarkeit von dt. -er und slaw. -nik weist auch das Nebeneinander der Formen Podawernik und Petawner (< slaw. *Pod-avorb-nikъ 'unter dem Ahorn wohnend, Unterahorner') im Musterregister aus 1385 hin. ${ }^{34}$

Insgesamt fand Bergmann in seinem Osttiroler Untersuchungsgebiet 21 Onyme, die das Suffix *-bnikb enthalten. Die slaw. Endung erscheint dabei im Dialekt in der Regel als [-ik], bei einzelnen Sprechern ist beim Verschlusslaut [-k] bisweilen eine Tendenz zur Affrizierung beobachtbar ([-ikx]), wohl in Analogie zu der auch in Osttiroler Mundarten nachweisbaren Auslautverhärtung von $-\mathrm{g} \mathrm{zu}[-\mathrm{kx}] .^{35}$

Weitere Beispiele: Ladinig (zu slow. ledina 'Brache'), Pototschnig / Petutschnig(g) (slow. Potočnik 'Bacher'), Glantschnig(g) / Quantschnig (slow. Kłančnik zu klanec 'Steile; Hohlweg'), an dt. Wortstämme tretend z.B. Kogelnig u. Freithofnig. ${ }^{36}$ In Osttirol kommen auch romanische Wortstämme vor, z.B. in Kals Rantschnigg (zu * runca 'Rodung' neben dem „dt." HN Rantschner und dem rom. Ranggetin(er)). ${ }^{37}$

3.3. -iče: Auch die SN auf -iče sind typisch alpenslawisch. Die in 2.2 genannten Namen Defereggen, Maßweg und Radweg / Radoviče gehören dem SN-Typus auf urslaw. *-itje $\mathrm{an}^{38}$. Dieser Namentyp bildet $\mathrm{SN}$ von PN, also Liebetig (Gem. Feldkirchen, 1433 Luebetikh < *L'ubotik'e zum PN L'ubota ${ }^{39}$ ), etwa 'Ort des L'ubota' bzw. 'Ort der Nachkommen des L'ubota' 40 . Eine Nebenform dazu ist die Kombination dieses Suffixes mit slaw. $-o v$ - $(-e v-)$, also ${ }^{*}$-ov-itje wie z.B. Maßweg < ${ }^{*}$ Měšovik'e zum PN *Měš $\left({ }^{*} M e ̌ s ̌ b k b\right){ }^{41}$. Beide Typen sind in den sogenannten "Kroatengauen“ besonders häufig (die beiden größten liegen im Bez. St. Veit a.d. Glan nördlich und südlich des Glantales in Kärnten und um Kraubath a.d. Mur im Bez. Knittelfeld in der Steiermark) ${ }^{42}$. Nach Miklosich ${ }^{43}$ kommt er nur im Westslawischen und westlichen Südslawischen vor, vgl. v.a. den skr. ON-Typus -(ov)ić ${ }^{44}$ (der v.a. im Zentrum des skr. Sprachgebietes häufig ist, an den Rändern, v.a. zum slow. Sprachgebiet hin, jedoch nicht ${ }^{45}$ ) und slow. -iče. Letzteres ist in SN im heutigen Österreich ungemein häufig, im heutigen Slowenien aber nicht ${ }^{46}$, einer der wenigen Namen ist z.B. Tupaliče (Gem. Kranj, 1154 Tupalich, 1377 Tupaelikch mit der auch in den alpenslaw. SN vorkommenden Lautung). Wir können also festhalten, dass slow. -iče für das Alpenslawische charakteristisch ist und jeder 20. Kärntner SN (also 5\%) hieher

\footnotetext{
34 vgl. Bergmann a.a.O.

35 vgl.. Kranzmayer 1956: 79.

36 dazu zuletzt Bergmann 2005: 150 und 200. - Bei Holzer 1991 nichts Vergleichbares.

37 vgl. Pohl 1998: 136.

38 -ištb bei Miklosich 1927: 118 (bzw. 1864: 2), poln. u. čech. -ice, slow. -iče (mit Apl. anstelle des Nominativs).

${ }^{39}$ Kronsteiner 1975b: 49 u. 1978: 150.

40 Miklosich a.a.O.

41 Kronsteiner 1975b: 51 u. 1978:153.

42 Dazu zuletzt Kronsteiner 1978: insb. 150ff. u. 153 (mit Karten).

43 1927: 118.

44 Nach Miklosich a.a.O. sei der auslautende Vokal sekundär abgefallen, doch nach Popović (1960: 439) kommen auch Formen wie -(ov)ići, $e$ vor.

45 Vgl. Popović 1960: 438, Karte 439 (mit Lit.).

46 Vgl. Bezlaj 1963: 88.
} 
gehört ${ }^{47}$. Ferner ist festzustellen, dass dieser Typus nur in jenen Gebieten vorkommt, die vor 1200 besiedelt worden sind ${ }^{48}$ - ein Hinweis darauf, dass in jenen Gegenden, in denen -iče vorkommt, die slow. Besiedlung spätestens im 12. Jhdt. erfolgt sein muss. Damit stimmt auch die Verbreitung dieses Typus in den 'Kroatengauen' (s.o.) überein, von denen erstmals jener um das Kärntner Glantal 954 als pagus Chrouuati genannt wird. Bei diesen 'Kroatengauen' handelt es sich ursprünglich um halbkreisförmig um einen zentralen Punkt (in unserem Fall um Faning, slow. Baniče, Gem. Moosburg, 1053 Vaniccha $<{ }^{*}$ Banik'e 'Ort eines ban, eines awarischen Fürsten') angeordnete Wehrsiedlungen ${ }^{49}$ und somit um einen der ältesten Zeugen alpenslawischer bzw. karantanischer Siedlungsnamengebung.

3.4. -vs, ...ves (vas): das alte slaw. Wort für 'Dorf' ist vasb, das in den deutschen Namensformen immer übersetzt wird; nur selten kommt urk. vbsb vor (in dt. oder lat. Kontext wie 993 Podinauuiz s.u.). Es entspricht dem lat. villa bzw. praedium, bezeichnet also den Einzelhof als Nukleus eines späteren Weilers (aus lat. villaris 'zum Gutshof gehörig'). Dieser Namentyp ist die „Leitform“ der Besiedlungsperiode bis $1100^{50}$. Daher hat sich vbsb offensichtlich nur im slaw. Westen „eingebürgert ${ }^{\text {“51. }}$. Daneben kommt aber dann auch se(d)lo 'Dorf' auf ${ }^{52}$, dieses bedeutete ursprünglich 'Siedlung' und kommt in Österreich relativ selten vor und ist von sedblo 'Sattel' nur schwer zu unterscheiden (s.o. 2.4.2). In den Fällen, wo zwischen dem Dental und $l$ ein Vokal erhalten ist bzw. urkundlich bezeugt ist, wird man wohl der Bedeutung

47 Kranzmayer 1956: 91.

48 Kranzmayer a.a.O.

49 Näheres vgl. Kronsteiner 1978: 148f. (mit Lit. u. Karten).

50 Vgl. Kranzmayer 1956: 88f. - trotz der Kritik von Kronsteiner 1975a: 14f. (mit Lit.) und Bezlaj 1963: 87. Dass se(d)lo das „ostslawische Wort für Dorf" sei (so Kranzmayer 1956: 87) ist freilich ein Irrtum.

51 So Bezlaj 1963: 87. Er weist allerdings Kranzmayers (1956:87f.) Behauptung zurück, wonach alle vbsbNamen auf bairisch-österreichischen Einfluss zurückzuführen seien. Doch deren überwiegende Mehrzahl liegt auf dem Boden des mittelalterlichen Römisch-Deutschen Reichs bzw. in dessen Vorfeld - so unrecht kann Kranzmayer also nicht haben.

52 Bemerkenswert erscheint die Tatsache, dass slaw. vbsb im Dt. mit 'Dorf', aber se(d)lo gewöhnlich mit Geschieß (auch Schuß) übersetzt wird (Kranzmayer 1956: 88 Anm. 33), Grundbedeutung 'Giebelmauer, wand' (Eberl 1925/26: 132 u. 178, von mhd. geschie3 'Giebelseite eines Gebäudes'), im dt. Süden offensichtlich auch 'Heim o.dgl.', z.B Račje selo 'Rapelgeschieß' (Gem. Trebnje/Treffen) und Ivanje selo 'Eibenschuß' (Gem. Cerknica/Zirknitz) im heutigen Slowenien. Auch in den nur das Grundwort se(d)lo enthaltenden SN kommt diese Übersetzung vor (in 4 von $33 \mathrm{SN}$ in der Südsteiermark/Štajersko [nach Blaznik 1988: 271ff.] bzw. 19 von 53 in Krain/Kranjsko [nach Kos 1975: 536ff.]). Aus Kärnten nennt Kranzmayer keine solchen Paare, mir sind derzeit auch keine bekannt. - Ein alter Kärntner SN Gschieß (Gem. Baldramsdorf) wurde in Rosenheim umbenannt (Kranzmayer 1958: 94).

53 Die Ablehnung von 'Sattel' seitens Bezlaj 1963: 79 und Kronsteiner 1975a: 14 („erst ab 1850“ in der Toponymie üblich) ist ebenso wenig berechtigt wie Kranzmayers (1958: 255f.) Bevorzugung dieses Appellativs gegenüber se(d)lo 'Dorf'. Es mag sein, dass 'Sattel' in neuerer Zeit wieder sehr populär geworden ist, dies schließt aber seinen Gebrauch im Mittelalter nicht aus. So sind z.B. in der ehemaligen Südsteiermark (heute Slowenien) 5 von insgesamt 8 in Frage kommenden slow. SN ins Dt. mit 'Sattel' übertragen belegt (Blaznik 1988: 269f.), in Krain/Kranjsko findet sich 1499 der Beleg Vsedli für eine Alm („planina pod Kamniškim sedlom", Steiner Alpen / Kamniške Alpe, Kos 1975: 536), aber keine 'Siedlung'. Ein in Kärnten in Frage kommender Ort (Zeltschach) hat als Parallele Sattelbogen neben sich (Kranzmayer 1958: 256). Außerdem nennt Eberl (1925/26: 162) ausdrücklich Sattel 'Bergsattel' als in der Toponymie übliches Appellativ. Somit ist weder an dt. Sattel noch an slow. sedzlo zu zweifeln. Vgl. auch den ukrain. BN Sidlo. 
'Sattel' den Vorzug geben müssen; in allen anderen Fällen ist von Name zu Name zu prüfen, ob 'Dorf, Siedlung' oder 'Sattel' anzunehmen ist ${ }^{53}$.

Nur zwei slowenische Namen mit vbsb sind früh belegt: Gösselsdorf / Goselna ves (1050: Goslauuis), sowie Podinauuiz (933) für heutiges Niederdorf (bei Hörzendorf). Sonst werden nur die deutschen Formen überliefert, z. B. 1106 Dobrendorf = Eberndorf / Dobrla ves (ma. Dobrolja bzw. Dobrilja ves, zum PN Dobrilo). Gerade dieses Beispiel zeigt, dass auch der deutsche Name nur aus dem Slowenischen her verständlich ist, aus *Dobrenja vbsb zu einem PN Dobren, halb übersetzt zu Dobrendorf, über Döberndorf mit „falsch“ abgetrenntem $d$ - (wohl vom Einwohnernamen *Döberndorfer $>{ }^{*}$ Déberndorfer mit missverstandenem Artikel, also uminterpretiert zu [(de) èbarndorfər] und weiter zu Öbern- bzw. Eberndorf (1060-70 Obrundorf). Vielfach stimmen diese Namen im Deutschen und Slowenischen nicht überein. Oftmals sind die diesen ON zugrunde liegenden PN unklar, da sie bis zur Unkenntlichkeit entstellt sind (wie z.B. in Loga ves / Augsdorf). ${ }^{54}$ Bei den meisten Namen handelt es sich um Ableitungen von PN, also dem Namen der Person, die mit der Gründung des Dorfes in irgendeiner Weise verbunden ist; sie liegen in der mittelalterlichen Großkolonisation (vor 1100) begründet und stellen einen ahd. Benennungstyp mit seiner slow. Entsprechung dar. Die sind alle nach dem selben Muster gebildet: im Deutschen sind sie Komposita, im Slowenischen Syntagmen aus einem PN + Possessivsuffix (meist $-j a$, fem. zu mask. Bildungen auf $-j i$ ) + ves (fem., ma. für vas), seltener andere Bildungen, z.B. Žamanje „Obersammelsdorf“, Lancova „Lanzendorf“. Nach E. Kranzmayer entsprechen einander über 90 Prozent der slow. ves-Namen bzw. 70 Prozent der dt. -dorf-Namen. In einigen slow. Namen ist das zweite Glied ves mit dem ersten zu einem Wort verbunden, z.B. Bilčovs „Ludmannsdorf“, wohl von den obliquen Kasus ausgehend (lautgesetzlich hätte der gemeinslaw. Nom.-Akk. vbsb, Gen.-Dat.-Lok. vbsi usw. slow. vas / ves, vsi usw. ergeben müssen und nach einem alten Gen. ${ }^{*}$ Biljče vsi, Lok. *(v) Biljči vsi wurde ein Akk. *Biljčo-vs gebildet, der dann die Basis für die heutige Namensform geliefert hat). Einen zweiten solchen Typ könnten Fälle wie Grabale ves „Grabalja ves / Grabelsdorf" und Dole ves „Dolnja ves / Unterdorf" (bei denen nur ves flektiert wird) repräsentieren.

\section{Lexikalisches (Skizze eines karantanischen Glossars)}

4.0. Die Symbiose zwischen den in die alpinen Gebiete Österreichs eingewanderten Slawen und den späteren Baiern war sehr eng. Die Eingliederung des (slawischen) karantanischen Fürstentums in das bairische Herrschaftsgebiet wirkte sich auch sprachlich aus. Einerseits setzten sich die bairisch-österreichischen Dialekte als lokale Verkehrssprachen immer mehr durch, andererseits hinterließ die slawische Grundschicht zahlreiche Wörter in der deutschen Mundart. Die meisten spezifisch „karantanischen“ Wörter sind Bergappellativa, doch daneben gibt es auch einige andere Wörter, hauptsächlich aus dem Bereich der bäuerlichen Sphäre (Pflanzen

54 vgl. Pohl 2000: 59 u. 110f.

55 auch Lehnübersetzungen wie Füchsling 'Eierschwammerl, Pfifferling' (wie slow. lisička zu lisica 'Fuchs', sonst sagt man in den bairisch-österreichischen Mundarten neben Eierschwammerl eher Rehling oder Reherl) - vgl. Pohl 2004: 67 u. 202. 
und Speisen). ${ }^{55}$ In der verklungenen alpenslawischen Sprache gab es auch deutsches Wortgut - beide Sprachen bereicherten einander also gegenseitig - wie dies in allen gemischtsprachigen Regionen zu beobachten ist. Als Beispiel sei hier der Wortschatz, der die bestehenden Herrschaftsstrukturen beschreibt und der den Kern der Untersuchung Holzers ${ }^{56}$ zur Sprache der Slawen in Österreich im Mittelalter darstellt. Die von ihm behandelten slawischen Wörter reflektieren zum Großteil speziell das Grundherrschaftswesen im bairischen Ostland. Die Wörter gehören verschiedenen Kategorien an, Entlehnungen aus dem Deutschen sind loss (Terminus der Landzuteilung, zu dt. Los $)^{57}$ und mytaŕb 'Mauteinnehmer' (zu mhd. mütaere), Lehnübersetzungen sind službba 'Dienst (servitium)', strělbcb 'Schütze', militärischer Terminus, geschrieben Strelz o.ä. und (vielleicht) poklonb 'Abgabe, Geschenk (eigentlich Verneigung ${ }^{58}$ )', geschrieben poklon, poglon, auch pogklann; ein slawisches Lehnwort im Bairischen ist županъ 'Vorsteher, Amtmann, Dorfrichter' (eigentlich 'Ältester'), ${ }^{59}$ eingedeutscht Suppan, eventuell auch einige andere. ${ }^{60}$ Aus dem karantanischen Bereich wäre auch noch ${ }^{*} k o s e z$ 'Edling (bevorrechteter Bauer, Freisasse)' zu erwähnen. ${ }^{61}$

Die nun folgende Liste erhebt keinen Anspruch auf Vollständigkeit ${ }^{62}$, vielmehr soll ein repräsentativer Querschnitt durch den älteren bairisch-österreichischen appellativischen Wortschatz slawischer Herkunft geboten werden, der die sprachgeographische Stellung des „Karantanischen" zwischen dem südlichen (dem Typ nach altslowenischen) und dem westlichen (dem heutigen Čechischen nahe stehenden) Slawischen illustriert. ${ }^{63}$

4.1. bbrdo '(felsiger) Berg, Anhöhe; Kamm' (gemeinslaw.): Pyhrn(pass), auch Pass Pyhrn, an der Grenze zwischen Oberösterreich und der Steiermark, traditionell richtige Aussprache [pirn] ${ }^{64}$ (1146 Pirdine, 1265 mons Pirn) $<{ }^{*} b_{b}$ bdina $^{65}$ (manche urkundliche Belege weisen auf das Grundwort, z.B. 1239 mons Pyrdo); in Osttirol:

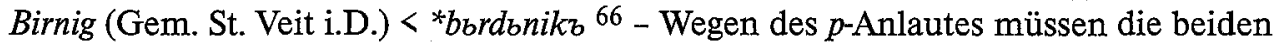

56 Holzer 2002 (alle Begriffe werden näher erläutert; hier sind nicht alle angeführt).

57 FN Losbnica (vgl. Holzer 2001: 75f.).

58 analog dt. Verehrung nach lat. honorarium (Holzer 2002: 64).

59 näheres s. Holzer 2001: $98 \mathrm{f}$.

60 vgl. Holzer 2002: 68.

61 zur Etymologie u.a. Bezlaj 1982: 69. Wohl türk.-awarischer Herkunft (so u.a. Kranzmayer 1956: 62f.), verwandt mit russ. kozak sowie türk. Kazach.

62 weitestgehende Vollständigheit weisen für ganz Österreich die Arbeiten von Müller 1971 und für Kärnten und Osttirol Pohl 1989a und 1990 sowie 1989b: 77ff. auf, weiters für Osttirol Bergmann 2002, für den Kärntner Küchenbereich Pohl 2004, für Bergappellativa Pohl 1999.

63 Auch das Slawische, wie es im Bereich des Erlaftales in Niederösterreich vorkommt, ist ein Übergangsdialekt zwischen dem Slowenischen und Čechischen (Holzer 2001: 119). Der südliche Teil dieses Gebietes dürfte jedenfalls zum karantanischen Fürstentum gehört haben, möglicherweise lag der Brettlbach (zu slaw. prédělb, s. 4.18) an dessen Nordgrenze.

64 nach Breu 1975: 227 (von Otto Back besorgt, vgl. S. XI); neuerdings setzt sich immer mehr die Aussprache [pYrn, püən o.ä.] durch, v.a. vom ORF verbreitet und auch vom „Österreichischen Wörterbuch" favorisiert (durch Erstnennung, 39. Auflage, S. 465).

65 Kronsteiner 1964: 70f.

66 Pohl 1996: 40.

67 im 8. Jhdt. nach Kranzmayer 1958: 24 sub Palten (mit weiteres Hinweisen), auch Kronsteiner 1975b: 148 bringt einige Beispiele für slaw. PN mit $p$. 
Belege sehr früh ins Deutsche entlehnt worden sein, ${ }^{67}$ denn später wird slaw. $b$ - durch ahd. $v>$ mhd./nhd. /f/ wiedergegeben, z.B. Verditz (K).

4.2. *chyša 'Hütte, Haus' (gemeinslaw.) $\rightarrow$ dt. Keusche: das aus german. *hūsa(dt. Haus) entlehne Wort bedeute auch im Slaw. 'Haus' und lautet im Slow. hiša. Es muss jedoch schön früh als Keusche 'kleines Bauernhaus, Achtelhube' rückentlehnt worden sein. Dies zeigt die alte slow. Entlehnung aus dem Deutschen (ma.) kaušlar 'Keuschler, Bewohner einer Keusche', das ein dt. *käusche voraussetzt (slaw. $y$ führt meist über $\bar{u}>\ddot{a} u \mathrm{zu} / a i /){ }^{68}$ Weiters zeigt die dt. ma. Nebenform khaiksn (St) bzw. Kaixen (Salzburg, Oberösterreich) bei der Entwicklung von slaw. $\check{s}$ die gleiche Entwicklung wie bei Kraxe (aus slaw. krošnja, 4.12, was sich auch in einigen ON findet (z.B. Trixen, slow. Trušnja) und gewisse Rückschlüsse auf die frühmdh. Aussprache des sch ziehen lässt. ${ }^{69}$

4.3. čblnъ 'Art Kahn' (gemeinslaw.) $\rightarrow$ dt. Zille, Zülle: die Kahnfahrt spielte bei den Slawen des Mittelalters eine große Rolle ${ }^{70}$; die deutsche Entsprechung kommt nur im östlichen Ober- und Mitteldeutschen vor. ${ }^{71}$

4.4. duplo 'Höhle', in dieser Form ein eher nach Süden weisendes Appellativ (kommt so im Čech. und Slowak. nicht vor, ${ }^{72}$ ist aber im Slow. u. Skr. häufig): Tauplitz $^{73}$ (St, SN/FN, Gem., GB Irdning, 1321 Tauplicz, 1425 Dewplitz [mit Umlaut] < *duplica), Teiplberg, -bach (St, GN/SN/BN, Gem. Lannach, GB Stainz, 14. Jhdt. Deupel [mit erhaltenem Umlaut] < * duplbje o.ä.).

4.5. dъbrb 'Schlucht, Klamm' (gemeinslaw.; in die Osttiroler Mundart als Daber / Daba entlehnt, in Ortsnamen in ganz Ost- u. Südostösterreich verbreitet): (Osttirol) Daberalm, -spitz (FN/BN), Daberalm, -kees, -kögele (FN/BN), Daberkees, -lenke (FN/GN), Dabernigalm (FN < HN Dabernig < *dъbronikъ 'der an der Schlucht, Klamm wohnt'), Dabernitzkees, -kogel (GN/BN < $\left.{ }^{*} d b b r b n i c a\right)^{74}$; (K) Dobratsch / Dobrač (Gailtaler Alpen, auch Villacher Alpe / Beljašcica, 1447 Dobritz $<{ }^{*}$ dobračb) ${ }^{75}$, Döbernitzen (SN, Gem. Kirchbach, Gailtal, 1374 Debernitz < ${ }^{*}$ dbbrbnica), Döbriach (SN, so seit 1369, Gem. Radenthein, 1117 Tibria < *dъbrjachъ, Lpl. des Einwohnernamens); (St) Dobrein (Gem. Mürzsteg, 1243 Tobryn < *dzbrina o.ä.), Tobergraben (Hochlantschgebiet, FN zum FN/SN Tober, Gem. Passail, 1240 Dobre, 1332 Tober) ${ }^{76}$.

4.6. gazъ (m.) und gazb (f.) (slow. gaz [m. u. f.] $]^{77}$ ) 'Über-, Durchgang; ausgetretener Weg durch den Schnee', prěgazı 'Übergang' (ein nur im Südslaw. vorkom-

\footnotetext{
68 vgl. Ramovš 1936: 37.

69 vgl. u.a. Lessiak 1910/1983: 260, zuletzt Holzer 2001: 54 Anm. 160 (mit weiteren Beispielen).

70 Holzer 2002: 389.

71 Kluge 2002: 1012 (anders Lessiak 1910/1983: 257f.).

72 nach Šmilauer 1970: 58.

73 Mader 1986: 98 bringt diesen Namen mit slaw. toplb 'warm' in Zusammenhang. Doch dort sind keine warmen Quellen o.dgl., vielmehr Sandsteinhöhlen, die namengebend waren, wie dies Finsterwalder 1971: 22 gezeigt hat.

74 alle Beispiele nach Pohl 1996b: 41.

75 Pohl 1985: 63.

76 Lochner 1997: 132. - Fern bleibt Dobra (NÖ), vgl. Bergermayer 2005: 60.

77 Pleteršnik II 208.

78 Vgl. ESSJa 6: 111f.
} 
mendes Wort, möglicherweise aus dem thrakischen Substrat ${ }^{78}$ ) in Pyhrgas (BN, Gem. Spital am Pyhrn, 1650 Pürgas, 1669/1762 Pir-/Pyrgas, vgl. den am Fuß des Berges liegenden FN Pyhrgasgatterl, Übergang vom Ennstal nach Norden, westlich an den Haller Mauern vorbei, nach Spital am Pyhrn bzw. Windischgarsten, daher Deutung 'Übergang' sehr wahrscheinlich ${ }^{79}$, eingedeutscht wohl noch vor der Liquidametathese, daher < *per-gazъ). In Kärnten gesellt sich der SN Wigasnitz, slow. Vijasce oder Vigasce, ma. Vigazice (Gem. Eisenkappel-Vellach) hinzu, wohl ein 'Ort, der große Teile des Jahres nur über Schneepfade erreicht werden kann'80.

4.7. glazb 'Stein, Fels, Rollstein' (kommt sonst nur im West- u. Ostslaw. sowie im Bulgarischen vor, daher ein mit dem Westslaw. gemeinsames Charakteristikum des Alpenslaw. wie u.a. der Erhalt der $d l$-Gruppe im Gailtaler Dialekt) in Graslitzen (BN, Gailtaler Alpen, 1524 Glasitzen, 1713-17 Clasitzen, Gläsitzen) < * glazica $^{81}$, weiters Glosbach (NÖ, Gem. Kilb) ${ }^{82}$.

4.8. gorica 'kleiner Berg, Bühel, Bichl' (dieses gemeinslaw. Wort, Diminutiv zu gora 'Berg', ist hinsichtlich seiner Bedeutung für den slow. u. skr. Sprachraum typisch; im Westslaw. steht es für 'Torf' oder 'Wäldchen'83; daher ist es kein Zufall, dass der nördlichste ON mit gorica im Süden von Niederösterreich liegt: Görtschach (GB Gloggnitz, 1297 de Gothsa, 1306 Gortsach, abgekommen, heute St. Christof, Gem. Prigglitz ${ }^{84}$ ), u.a. in Salzburg Gerzkopf (BN, Gem. St. Martin im Pongau) oder in der Steiermark Göritz (heute SN, Gem. Parschlug, Bruck a.d. Mur; 1307, 1353 Goeritz), desgleichen Göritz in Oberösterreich (SN, Gem. Nußbach, Bez. Kirchdorf, 1299 Goertz, und BN im Tal der Krummen Steyrling 1531 Göritzstain, 1787 -berg). In Kärnten v.a. als SN (Einwohnername im Lpl.) Görtschach (5x) bzw. Goritschach (10x), im Slow. Goriče (insg. 9x).

4.9. grič̌ 'Hügel, Steile, Anhöhe' (alte, nur im Südslaw. begegnende Ableitung von gora ${ }^{85}$ ): Gritsch (BN, Gem. Assling, Osttirol), Gritschbühel (BN, Gem. Zederhaus, Salzburg), Gritschenberg (Gem. Niederöblarn, St, 1341 Gritschenperg), Gritschenhöhe (Gem. St. Kathrein am Hauenstein, St, ca. 1600 Gritschenberg, HN Gritsch).

4.10. južina 'Mittagessen' (gemeinslaw.) $\rightarrow$ dt. Jause 'kleiner Imbiss, Zwischenmahlzeit'; zusammen mit Jausenstation ist dieses Wort zu einem Parade-Austriazismus geworden. Entlehnt aus slow. južina 'Mittagessen', welche Bedeutung Jause heute noch im Kärntner Lesachtal hat; die Jause selbst wird im Kärntner Slowenischen (heute) mala južina 'kleines Mittagessen' genannt, in Kärnten meist [máwžna] ausgesprochen. Das Unterbleiben des Umlautes im Deutschen wird durch die Nebenform slow. južna erklärt ${ }^{86}$, doch lokal kommt Umlaut vor, z.B. in der

\footnotetext{
79 so Lochner 1985: 12, mit Vorbehalt Kronsteiner 1980: 225.

80 Kranzmayer 1958: 245.

81 Pohl 1985: 65, zuletzt Pohl 2002b: 75.

82 Bergermayer 2005: $79 f$.

83 ESSJa 7: 45.

84 Bergermayer 2005: 83.

$85 \mathrm{Vgl}$. ESSJa 7:128.

86 Pleteršnik I 375, Steinhauser 1978: 122

87 Hornung 2002: 521.

88 vgl. Steinhauser 1978: 120 .
} 
Gottschee / Kočevje (Jaischn ${ }^{87}$ ). Eine Entlehnung aus dem Čech. oder Slowak. kommt aus lautgeschichtlichen und sachlichen Gründen nicht in Frage. ${ }^{88}$

4.11. koper 'Dill, Gurkenkraut, (auch) Kamille, Bärenwurz' (gemeinslaw.) $\rightarrow$ dt. Koper und koprc 'Fenchel; Sophienrauke': In Kärnten ist Koper ein gängiges Wort für den Dill, ${ }^{89}$ koprc findet sich als Göpritz in Osttirol mit spezialisierter Bedeutung, z.B. 'Madaun (Ligusticum mutellina)', dessen Blätter tatsächlich an die des Dills erinnern ${ }^{90}$, ähnlich ein Almfutterkraut in der Lautung [kuprits] in Oberkärnten (Mölltal). ${ }^{91}$

4.12. *krosno ${ }^{92}$ neben *krosńa 'Rückentrage (meist aus Holz, auch geflochten als Korb)' (gemeinslaw.) $\rightarrow$ Kraxe (Krächse): früh entlehnt noch in der alten slaw. Lautung *krăšna $a{ }^{93}$ dem Vorgänger von slow. krošnja (da das Wort im Čech. krosna lautet, muss es altslowenischer Herkunft sein $\left.{ }^{94}\right) .{ }^{95}$ Zur Wiedergabe des slaw. $\breve{s}$ mit chs s.o. 4.2; frühslaw. $\breve{a}$ statt $o$ findet sich auch in einigen anderen frühen Entlehnungen, ${ }^{96} \mathrm{vgl}$. z.B. den urkundlichen Beleg Astaruuiza für heutiges Hoch-osterwitz.

4.13. kyselica 'Säuerliches' (gemeinslaw.) $\rightarrow$ dt. Gaislitz: Die alte bäuerliche Speise Gaislitz findet sich bei Lexer ${ }^{97}$ unter dem Eintrag geislaz, -liz (m.) bzw. geislazn (f.) 'Speise aus Hafermehl' (Möll-, Drautal, Unterkärnten). Eine genaue Beschreibung dieser Speise bietet Hornung 98 für Kals (Osttirol), wo diese Speise gāi(z)litß lautet und ein saurer, fettloser Haferbrei ist, der in erstarrtem Zustand kalt gegessen wird. Auch hier haben wir ein altes slawisches Lehnwort vor uns, das schon in mittelhochdeutscher Zeit belegt ist: gîs(e)litz(e) (m., f.) 'breiartige Speise ${ }^{99}$ und auf slaw. ${ }^{*}$ kyselica beruht ${ }^{100}$. Im Stift St. Florian bei Linz wird diese Speise schon im 12. Jhdt. als giselitz zitiert ${ }^{101}$ und scheint weit verbreitet gewesen zu sein. Wie bei den Tålggen gibt es auch bei dieser Speise einen Bezug zum Russischen. Dort wird zwar nicht die Form *kyselica verwendet, sondern kisél' (aus *kyselb), was nach dem Dahl'schen Wörterbuch ein Brei aus Hafer, Gerste und Weizenmehl ist, der warm gestellt und gesäuert wird. Urkundlich ist diese Speise bereits 997 belegt ${ }^{102}$.

Bemerkenswert erscheint die Tatsache, dass die Wörter (und Sachen) Tålggen, Munggen und Gaislitz zwar eindeutig slawischer Herkunft sind, aber bei unseren

89 vgl. u.a. Pohl 2000: 362.

90 Bergmann 2005: 105.

91 Hornung 1973: 112.

92 so ESSJa $13: 13$.

93 Holzer (2001: 54 Anm. 160) setzt *krašnjaa an.

94 Steinhauser 1978: 126 u. Anm. 530 (mit Lit.), weiters vgl. Bezlaj 1982: 100, Snoj 1997: 278.

95 vgl. auch Pohl 1989: 259 mit Lit.

96 weitere Beispiele bei Ramovš 1936: 39.

97 Lexer 1862: 112.

98 Hornung 1964: 79.

99 Lexer 1983: 73. Auch bei Schmeller (I/2:952) als die Geislitze 'eine geringe Speise' / der Geislitz 'Haferbrei' enthalten.

100 im südbairischen Raum eher aus altem slow. kiselica (heute meist 'Sauerampfer'), in anderen Gegenden auch aus tschech. kyselice 'Säuerliches' herleitbar (wofür sich Hornung 1964:79 ausspricht).

101 vgl. Rhamm 1909: 215.

102 Rhamm 1909: 220. 
heutigen slawischen Nachbarn nicht mehr vorkommen, wohl aber bei den Russen. Daraus kann man schließen, dass bei den alten Slawen der Hafer eine sehr große Rolle gespielt hat, was bei den Russen (und Finnen) im Osten und bei den Alpenslawen im Westen noch lange erhalten geblieben ist, auch zu einer Zeit, als man in den Alpen schon längst deutsch sprach. Daher sind die Lautformen dieser Speisen auch aus slawistischer Sicht sehr altertümlich und sie werfen ein interessantes Licht auf die Kulturgeschichte des Essens.

4.14. lanež (slow.) 'Bergrücken, Kamm, Grat (der schwer zu übersteigen ist)'103. Die Herkunft des Wortes aus einer Ableitung von slow. lani 'voriges Jahr' erscheint möglich, vgl. das Paar dt. Ferner rom. vedretta, beide 'Altschnee', von lat. vetus 'alt'104. Das dt. Ferner hängt mit Firn 'vorjähriger Schnee', mhd. virne, vern(et) 'im vorigen Jahr' zusammen, vgl. auch bair. ferten 'ds.'. Es könnte also eine südalpine rom.-dt.-slow. semantische Gleichung vorliegen wie dies u.a. auch bei 'Dachboden' (südbair. Unterdåch, slow. podstrešje, furl. sotèt rom. subtum tectum 'unter dem Dach') und 'Frühling' (südbair. Auswart, slow. ma. vigred, furl. inšude rom. (*in-) exitus 'Ausgang') der Fall ist. Wenn diese hier vorgeschlagene Deutung richtig ist, wäre die Grundbedeutung von slow. lanež 'mit Firn überzogener Bergrücken oder Kamm, Gletscher', die sich mit dem Rückgang der Vergletscherung zu 'schwer zu überwindender Bergrücken usw.' gewandelt hat. Das Appellativ kommt auch zwei Mal in Slowenien in den Kamniške in Savinjske Alpe / Steiner und Sanntaler Alpen vor, wo man sich vorstellen kann, dass bei kälterem Klima der Firn lange liegen blieb. Zwei Namen in Kärnten: Wainasch / Vajnež (BN, Karawanken, wohl aus *lanjež > ma. łajnāžz̆ [wájnəž] ${ }^{105}$ ) und Lanischeck, -kees (BN/GN, Ankogelgruppe).

4.15. mqka 'Mehl' (gemeinslaw.) und *tălkŭnă 'Hafermehl' (west- u. ostslaw.?) $\rightarrow$ dt. Munggen und Talggen: Unter diesen beiden Bezeichnungen versteht man ein uraltes bäuerliches Vollkornnahrungsmittel ${ }^{106}$ - mit Hafer. Lexer verzeichnet diese beiden Wörter als talk, talgge $\mathrm{m}$. 'Hafergrütze' (Drautal) ${ }^{107}$ und munkn, munggn $\mathrm{f}$. 'eine Nationalspeise aus Hafer- und Gerstenmehl; (auch) kleines Stück Brot (Feldkirchen)' ${ }^{108}$. Hornung ${ }^{109}$ verzeichnet die Munggn als Mölltaler Frühstücksspeise, genauer werden von ihr aber beide Wörter, die im Grunde genommen dieselbe Speise bezeichnen, so ${ }^{110}$ beschrieben: Munkn 'Altkärntner Nationalspeise aus Hafer, Gersten- und Bohnenschrot, der gekocht, getrocknet und dann gemahlen aufbewahrt wird, um fallweise mit heißem Wasser angerührt zu werden'; Tålggn 'Hafer-, Gersten- und Bohnenschrot, gekocht, getrocknet und dann gemahlen', auch für zerkleinerte und getrocknete Birnen verwendet. Man findet in Kärnten den Ausdruck Talggen v.a. im Nockgebiet, Munggen im Lesach- und Mölltal.111

\footnotetext{
103 so Badjura 1953:75, er nennt auch lanževica und bedauert, dass dieses Wort bei Pleteršnik fehlt.

104 Kranzmayer 1968:11.

105 vgl. bei Pleteršnik I 499 Formen wie lanjski 'vorjährig', lanjščak 'vorjähriges (Haus-) Tier' usw.

106 vgl. Pohl 2004: 32ff. mit Lit.

107 Lexer 1862: 51.

108 Lexer 1862: 193.

109 Hornung 1968: 181.

110 Hornung 1966: 174 u. 176.

111 Näheres zu diesen Speisen zuletzt Pohl 2004: 32ff.
} 
Beide Wörter, Munggen wie Talggen, sind aus dem frühen Mittelalter überkommene Wörter. Das Wort Munggen ist ein frühslowenisches Lehnwort (aus slaw. *moka 'Mehl', slow. moka). Ein ebenso hohes Alter müssen die Talggen haben, die auf einem urslaw. *tălkŭnă (woraus russ. toloknó 'Art Hafergrütze', ins Finnische als talkkuna entlehnt, schon im finnischen Nationalepos Kalevala bezeugt ${ }^{112}$ ) beruhen, was ein frühslow. ${ }^{\star}$ talkno bzw. slow. ${ }^{*}$ tlakno (vgl. poln. tłokno 'Speise aus Hafermehl, heißem Wasser und Milch') ergeben hätte müssen. ${ }^{113}$ Wahrscheinlich handelt es sich um ein slawisches Erbwort, zur Wurzel *tălk- 'stoßen, (zer)stampfen' in russ. toloc'/tolkat', zur Bedeutung vgl. auch im Keltischen u.a. kymr. („Welsh“) talch 'granum contritum' ('geschrotetes/gemahlenes Korn') bzw. 'Bruchstück, Mahlkorn', altkorn. talch 'Kleie'114. Das Wort muss also sehr früh ins Deutsche gelangt sein (spätestens zur Zeit der sogenannten slawischen "Liquidametathese“, die in den slawischen Einzelsprachen zur Umstellung von ${ }^{*}$ tălk- zu tlak-/tlok-/tolokgeführt hat, also etwa im 9. Jhdt.). Die Talggen sind in Österreich weit verbreitet, außer in Kärnten und Osttirol auch im Salzburger Lungau und Teilen der Steiermark (s.a. 4.13). - Davon zu trennen sind allerdings die Wiener (auch nieder- und oberösterreichischen) Dalken aus Germteig („Böhmische Dalken“) ${ }^{115}$.

4.16. oblica 'Rübe' (slow.) $\rightarrow$ dt. Oblitzen: Ein weiteres slowenisches Reliktwort in Oberkärnten und Osttirol (Defereggen-, Iseltal) ist Oblitzen 'weiße Rübe' (aus slow. ma. oblica 'gesottene oder gebratene Rübe') ${ }^{116}$.

4.17. pograd 'Gerüst an der Wand, das als Gestell dient; Pritsche' (slow.) $\rightarrow$ dt. Pograte (f.) ${ }^{117}$ : ins Deutsche entlehnt meist mit der Bedeutung 'Gemeinschaftslager in Holzknecht- und Almhütten; einfache Schlafstelle; erhöhte Abstellfläche, Zwischenboden'; verbreitet v.a. im Südosten Österreichs. Wie alt die Entlehnung ist, lässt sich nicht feststellen, aber da das Wort auch in der seit dem 13. Jhdt bestehenden, von Osttirol aus besiedelten Sprachinsel Pladen / Sappada vorkommt ${ }^{118}$, muss dies relativ früh erfolgt sein ${ }^{119}$.

\section{Rhamm 1909: 219.}

113 worauf schon Ramovš 1936: 25 hinweist.

114 vgl. Pohl 2004: 34 mit Lit. Nach Kranzmayer 1949: 459, zuletzt WBMÖ IV 506, stamme das Wort letzten Endes aus dem Mongolischen und sei über die Awaren in unseren Bereich gelangt, was man zwar nicht ganz ausschließen kann, aber für diese frühe Zeit nicht sehr wahrscheinlich und darüber hinaus lautgeschichtlich bedenklich ist, wie übrigens auch die Ansicht, dass ungar. tarhonya 'Art Teigreis, Eiergraupen' damit zusammenhängt (Kranzmayer 1949: 460, Rhamm 1909: 221 Anm.).

115 die auf einem anderen slaw. Wort beruhen (tschech. dolek 'Vertiefung'), vgl. Kranzmayer 1949: 459, WBMÖ IV 505f., zuletzt Hornung 2002: 221. - Beide Wörter vermengt bei Schmeller I/1: 505.

116 vgl. Hornung 1964: 77 u. 159, Lexer 1862: 200.

117 WBMÖ III 559 (Varianten Pogate, Grat).

118 vgl. Hornung 1992: 106f.

119 bevor die ersten slowenischen Holzarbeiter das Wort auch nach Salzburg (ins Salzkammergut) brachten (vgl. Bezlaj 1995: 74).

120 so Holzer 1991: 50.

121 Šmilauer 1970: 54, Badjura 1953: 105. - Nicht immer leicht zu trennen von: dolb 'Tal', dolina 'breites Tal' (Šmilauer 1970: 54, Badjura 1953: 204f.), prědolb 'Pass, Bergübergang (wörtl. Zwischental)' (s.o. (prě)dèlı; kommt auch in SN vor, z.B. Dolina u. Dellach, mehrmals K), z.B. Dolinzaalpe (K) GN Dolinzabach < *dolinica (Pohl 2001: 52); Pridou/Predol (K, Pohl 1984, 36); Pretul(alpe) (St, 1289 Predili; Kronsteiner 1964: 68); Pretal-(Mader 1986, 77).

122 Steinhauser 1932: 9 
4.18. prěděl` 'Wasserscheide ${ }^{120}$, Pass' (zu gemeinslaw. dělø 'Berg(rücken), Gebirgszug'121, kommt auch in SN vor, z.B. Predl, K, und Predlitz, St, bekannter BN außerhalb Österreichs Predil [SLO/I]), z.B. Brettl (N, 1220-40 Predel $\left.{ }^{122}\right) ;$ Pretal (St), Pretalsattel, -berg (St, Predal ${ }^{123}$ ). Das Wort prědel konnte oft auch 'Grenze' bedeuten und markierte 5 oder 6 Mal die Grenzen des alten Karantanien (Brettl(bach), Predil, Prietal usw. $)^{124}$.

4.19. * $\mathrm{p}(\mathrm{r})$ ętro (n.) bzw. * $\mathrm{p}(\mathrm{r})$ ętra (f.) 'Art Gestell aus Brettern' (gemeinslaw.) $\rightarrow$ dt. P(r)änter, Gepäter usw. ${ }^{125}$, im Slow. petre (f.pl.) 'Brettergerüst zur Aufbewahrung von Stroh oder Heu in Stallungen, Scheunen u.dgl.', petro 'Heuboden'126, in Kärnten ma. peter $(\mathrm{m}$.$) 'Oberboden der Scheune { }^{122}$, patar / patra' ${ }^{128}$ (auch in anderen slaw. Sprachen belegbar, z.B. čech., slowak. patro, osorb. pratr) ${ }^{129}$. Dieses (v.a. im Südbairischen - ohne Nord- und Südtirol - verbreitete) Wort ist zu der Zeit ins Deutsche entlehnt worden, als der Nasalvokal noch nicht vollständig geschwunden war, denn wir finden Formen mit und ohne - $n$-, die Bedeutung stimmt mit der heutigen slowenischen überein. Die häufigsten Varianten sind Pater, Gepater, auch Gepranter (meist mit [a], selten [å]).

4.20. stodorb 'Felsgrund, (dürftiger und seichter) Ackerboden auf felsigem Grund; steil aufragender, felsiger Berg' (bes. verbreitet im Alpenraum, aber auch im Westslaw. ${ }^{130}$ ): Stoder(tal) (SN, Gem., Oberösterreich, 1467 im Stoder, heute: Vorder/Hinterstoder), hier liegt die erste Bedeutung zugrunde ${ }^{131}$; Stador (BN, Gailtaler Alpen), Gstoder [štóudə] (BN, Salzburg / St), Stoderzinken (BN, Gem. Gröbming, St), Stadurz (BN, Gem. Wildalpen, St, 1454-64 Staducz < ${ }^{*}$ stodorbcb).

4.21. *strąkb (m.) bzw. *strąka (f.) 'Schote' $\rightarrow$ dt. Strankerl (genauer Stränggelein) [štránkəle, Plural -lan] grüne Bohne, Fisole (Phaesolus vulgaris); typisch für Kärnten, hier allgemein üblich, auch in der geschriebenen Sprache, < frühslow. *strakb (bzw. *strąka) '(Bohnen-) Schote', modern slow. strok 'Hülse, Schote'. Sehr früh entlehnt, jedenfalls früher als Munggen (s. 4.15), das noch den Lautwandel urslaw. / frühslow. $a \mathrm{zu}$ altslow. Q mitgemacht hat. Die heute allein übliche Diminutivform setzt ein deutsches mundartliches Grundwort *štrånka 'Bohnenschote' voraus ${ }^{132}$, dem

123 Kronsteiner 1964: 67, Lochner 1985: 21

124 Holzer 1997: 94f., zuletzt 2001: 50, Bergermayer 2005: 194. - Im Bairischen entspricht dem slow. prědělı das Appellativ Gschaid (zu scheiden, vgl. u.a. Holzer 2001: 50 mit Lit.), einmal als Grenzpunkt Karantaniens bzw. seines Nachfolgers, des alten Herzogtums Kärnten, belegt: Karintscheide (später dann Landesgrenze zur Steiermark, s. ANB I 582). Einen weiteren Grenzpunkt markiert der Pockaubach (um 1000 in Paca, 1352 aqua Poka), der semantisch eine Wasserscheide bezeichnet (Holzer 2001: 86, ANB I 122).

125 Ansätze nach WBMÖ II $245 f$.

126 Pleteršnik II $32 \mathrm{f}$.

127 Karničar 1990: 207.

128 Zdovc 1972: 71 u. 80.

129 nähere Angaben Bezlaj 1995: 32.

130 Vgl. Bezlaj 1995: 338 s.v. studor.

131 Finsterwalder 1971: 26 mit Lit.

132 vgl. Lessiak 1903: 153. - An Versuchen, das Wort german. zu deuten, hat es nicht gefehit (u.a. bei Lexer 1862: 243 u. Schmeller II/1: 817, die es mit deutsch Strang in Zusammenhang brachten). 
Synonym von Strankerl, etwa [póanšādl], liegt jedoch Bohn(en)scheidel (und nicht schote) zugrunde.

4.22. *tălkŭnă s. moka (4.15)

4.23. 'topenica 'Erwärmtes, Erhitztes' (gemeinslaw.) $\rightarrow$ dt. Topanitz: ein altes slaw. Reliktwort, u.a. in Kals (Osttirol), eine Speise, die Hornung ${ }^{133}$ als wenig schmackhafte trockene Bähschnitten (Art Toast) beschreibt und was auf ein slaw. *topenica (zu topel 'warm', topiti 'erwärmen, zum Schmelzen bringen (von Metall); (gemeinslaw. auch) heizen', im Slowak. topenica) zurückgehen dürfte. ${ }^{134}$

\section{Zusammenfassung}

Sprachgeographisch steht das Slawische des ehemaligen karantanischen Fürstentums zwischen dem Vorläufer des (heutigen) Slowenischen im Süden und dem des (heutigen) Čechischen im Norden, stellt also dialektologisch ein Übergangsgebiet zwischen dem slawischen Nordwesten und Südwesten dar, worauf u.a. die weit in den Süden reichende $d l$-Isoglosse (2.4) hindeutet sowie einige Lexeme, die teils für den Norden (z.B. glazb, 4.7), teils aber auch für den Süden (z.B. gričcb, 4.9) charakteristisch sind. Zu ähnlichen Schlüssen kommt auch Holzer ${ }^{135}$ bezüglich der Slavia submersa im Erlaftal (NÖ), die sich v.a. in einem Punkt jedoch deutlich von unserem Untersuchungsgebiet abhebt: während im Erlaftal die Entwicklung von urslaw. ${ }^{*} t j>$ $c$ führt, ist im „Karantanischen“ das Ergebnis zunächst $t$ ' bzw. $k$, im Deutschen in der Regel durch /k/ wiedergegeben, z.B. Lonitz (NÖ, < ${ }^{*}$ Lochynici) ${ }^{136}$ gegenüber Maßweg (St, < *Měšovik'e, 2.2 u. 3.3), 'später dann slow. $\check{c}$ (z.B. Radweg/Radoviče, K).

Es erscheint also berechtigt, von einem karantanischen Slawisch zu sprechen, das sich im Laufe des Frühmittelalters als Übergang zwischen dem späteren Čechischen im Norden und Slowenischen im Süden herausgebildet hat. Viele altertümliche Formen kommen jenem Sprachzustand nahe, den man „urslawisch" nennt (z.B. kurzes $\breve{a}$ statt $o, 4.12$ oder Unterbleiben der Liquidametathese, 4.22), doch im Laufe der Zeit nimmt das Sprachmaterial (im Süden zunehmend) immer mehr die Züge an, wie sie für die heutigen Dialekte Kärntens typisch sind. In (relativ) früh eingedeutschten Gebieten haben sich vielfach alte (urslawische) Formen erhalten (z.B. das Fehlen der $j$-Prothese, 2.5). Dieses „Karantanisch“ entspricht im Großen und Ganzen gesehen zwar dem traditionellen „Alpenslawischen“ (nach Ramovš), grenzt es aber dennoch auf das alte Karantanien ein. Literarisch ist dieses Karantanisch in den altslowenischen „Freisinger Denkmälern / Brižinski spomeniki“ überliefert, zu deren Sprache die alten slowenischen Lehnwörter im Deutschen und das Namengut slawischer Herkunft eine auffallende Affinität haben. Daher ist das "Karantanische" wie auch das Erlaftaler Slawische und Plattenseeslawische ${ }^{137}$ einer der gemeinslawischen Dialekte des Mittelalters, dessen Südrand später in die

133 Hornung 1964: 79.

134 Müller 1971: 173

135 2001: 119 .

136 Holzer 2001: 74.

137 vgl. Holzer 2001: 119 mit Lit. 
slowenische Glotto- und Ethnogenese einbezogen worden ist, wobei sich einige Eigenheiten bis heute bewahrt haben.

\section{Literatur}

ANB: Altdeutsches Namenbuch. Die Überlieferung der Ortsnamen in Österreich und Südtirol von den Anfängen bis 1200 . Wien $1989 \mathrm{ff}$.

BADJURA, R.: Ljudska geografija. Ljubljana 1953.

Bergermayer, A: Glossar der eingedeutschten Namen slavischer Herkunft in Niederösterreich. Wien 2005.

BergmanN, H.: Slawisches im Namengut der Osttiroler Gemeinden Ainet und Schlaiten. Anmerkungen zur Slavia Submersa im vorderen Iseltal. Dissertation Klagenfurt 2003.

Bergmann, H.: Mundart und Name in Osttirol. In: Tirol an Isel und Drau. Eine Annäherung. Schlanders 2005.

BEZLAJ, F.: Etimološki slovar slovenskega jezika I-III. Ljublana 1976, 1982, 1995.

BLAZNIK, P.: Historična topografija slovenske Štajerske in jugoslovanskega dela Koroške do leta 1500. Maribor 1986-1989 (3. Bde.).

BRANDENSTEIN, W: Kleine namenkundliche Arbeiten. Graz 1978.

BREU, J.: Geographisches Namebuch Österreichs. Wien 1975.

CVETKO-OREŠNIK, V.: Slovenskost Brižinskih spomenikov in nekatere novejše hipoteze o njih. In: Obdobja 10 (1989) 35-50.

CVETKO-OREŠNIK, V.: K Isačenkovemu poskusu zavrnitve Ramovševih argumentov za slovenskost Brižinskih spomenikov. In: Zbornik Brižinski spomeniki. Ljubljana 1996, 193-202.

EBERL, B.: Die bayerischen Ortsnamen I 2. München 1925/26.

ESSJ $_{A}=$ Etimologičeskij slovar' slavjanskich jazykov. Moskva $1974 \mathrm{ff}$.

FINSTERWALDER, K.: Um Tauplitz und Mölbing. In: Jahrbuch des Österreichischen Alpenvereins 1971, $19-28$.

FINSTERWALDER, K.: Tiroler Ortsnamenkunde I-III. Innsbruck 1990-1995.

Holzer, G.: Die Herkunft des $(t) z$ in Kollmitz etc. ( ${ }^{*}$ kalamantiā), Kobenz (*kumbantiā) und Lafnitz (*albantiā), in: Österreichische Namenforschung 25 (1997 = Festschrift Odwarka) 81-103.

Holzer, G.: Die Slaven im Erlaftal. Eine Namenlandschaft in Niederösterreich. Wien 2001.

Holzer, G.: Zur Sprache des mittelalterlichen Slaventums in Österreich. Slavisch unter bairischem Einfluss. In: Wiener Slavistisches Jahrbuch 48 (2002) 53-73 (= 2002a).

Holzer, G.: O leksiku slavenskoga supstrata u Austriji. II. Pregled o građi. In: Folia onomastica Croatica 11 (2002) 95-102 (= 2002b).

Holzer, G.: Landschaft und Siedlung im slavischen Frühmittelalter. In: Namen, Sprachen und Kulturen (Festschrift für H.D. Pohl). Wien 2002, 387-397 (= 2002c).

HörBURGER, F.: Salzburger Ortsnamenbuch. Salzburg 1982.

HoRNung, M., Mundartkunde Osttirols. Wien, ÖAW 1964.

HoRnung, M., Mundartwörterverzeichnis. In: Dinklage, Karl (Hrsg. et alii), Geschichte der Kärntner Landwirtschaft. Klagenfurt 1966, S. 169-177.

Hornung, M., Die Mundart des oberen und mittleren Mölltales. In: Prasch, H., Um die Möll. Spittal/Drau o.J. [1968], S. 81-84 (Beschreibung) u. 179-182 (Wörterverzeichnis).

HoRNUNG, M.: Beobachtungen über die kategorienbildende Funktion slawischer Suffixe bei deutschem Namenmaterial in Sprachberührungszonen. In: Onomastica Slavogermanica 13(1981) 61-66.

HoRNUNG, M., Dialektgeographische Raumgestaltung im oberen und mittleren Mölltal. In: Zeitschrift für Dialektologie und Linguistik, Beiheft 9 (1973) = Festschrift Kurath 107-117.

HoRNUNG, M., Wörterbuch der Wiener Mundart. Wien, ÖBV 1998 (2. Auflage 2002).

KARNICAR, L.., Der Obir-Dialekt in Kärnten. Die Mundart von Ebriach / Obirsko. Wien 1990.

KlUGE, F., Etymologisches Wörterbuch der deutschen Sprache (23. Auflage, bearbeitet von Elmar Seebold). Berlin-New York, Walter de Gruyter 1999 (24. Auflage 2002).

Kos, M.: Gradivo za historično topografijo Slovenije (za Kranjsko do leta 1500). Ljubljana 1975, 3 Bde.

KRANZMAYER, E.: Kärntner Bauernkost und ihre Geschichte. In: Carinthia I 139 (1949), S. 446-462.

KRANZMAYER, E.: Ortsnamenbuch von Kärnten I-II. Klagenfurt 1956-1958.

KRANZMAYER, E.: Kleine namenkundliche Schriften. Wien 1997.

KRONSTEINER, O.: Slawische Elemente in den Bergnamen der Steiermark. Wien 1964 (Diss.).

KRONSTEINER, O.: Edlitz, Selzthal, Zell und Zeltschach. In: Österreichische Namenforschung 1975/1, 12-21 (= 1975a).

Kronsteiner, O.: Die alpenslawischen Personennamen. Wien 1975 (= 1975b).

KRONSTEINER, O.: Gab es unter den Alpenslawen eine kroatische ethnische Gruppe? In: Wiener slavistisches Jahrbuch 24(1978) 137-157 (nachgedruckt in: Österreichische Namenforschung 1978 Beilage). 
KRONSTEINER, O.: Die slawischen Ortsnamen in Oberösterreich. In: Baiern und Slawen in Oberösterreich (red. v. K. Holter), Linz 1980, 211-228.

KronsteIner, O., Die slowenischen Namen Kärntens (mit einer Einleitung von H.D. Pohl). Wien 1982 (3. veränderte und erweiterte Auflage).

LESSIAK, P.: Alpendeutsche und Alpenslawen in ihren sprachlichen Beziehungen. In: Germanisch-Romanische Monatsschrift 2 (1910) 277-288, nachgedruckt in: Die Wiener dialektologische Schule (hg. v. P. Wiesinger), Wien 1983, 249-263.

LEXER, M., Kärntisches Wörterbuch. Leipzig 1862.

LOCHNER VON HÜtTENBACH, F.: Bergnamen in der Steiermark. Graz 1985.

LOCHNER VON HÜTTENBACH, F.: Zur Namenkunde des Hochlantschgebietes. In: Österreichische Namenforschung 25(1997) 131-136.

MADER, B.: Die Alpenslawen in der Steiermark. Wien 1986.

MikLosich, F.: Die Bildung der Ortsnamen aus Personennamen im Slavischen. Wien 1864.

Mikiosich, F.: Die Bildung der slavischen Personen- und Ortsnamen. Heidelberg 1927.

Müller, B.: Zur Typisierung des Einflusses slawischer Sprachen auf den Wortschatz der deutschen Sprache dargestellt am Beispiel des Bairisch-Österreichischen. Dissertation Berlin 1971.

NewEKLowsKY, G.: 224. Deutsch-Kroatisch. In: In: Kontaktlinguistik. Ein internationales Handbuch zeitgenössischer Forschung, 2. Halbband. Berlin-New York 1997, 1821-1827.

ODWARKA, K. - Pohl, H.D.: Materialien zu einem Namenbuch von Kals (Osttiro1), 5. Teil: Hofnamen. In: Österreichische Namenforschung 26 (1998) 1-2, 124-145.

PLETERŠNIK, M., Slovensko-nemški slovar I-II. Ljubljana 1894 (Nachdruck 1974).

POHL, H.D.: Wörterbuch der Bergnamen Österreichs I. Kurzgefaßtes Verzeichnis der österreichischen Bergnamen. Salzburg 1984 ( $=1984 \mathrm{a})$.

PoHL, H.D.: Kärntner Bergnamen II. In: Österreichische Namenforschung 12 (1984) 1, $25-45$ (1984b).

POHL, H.D.: Kärntner Bergnamen III. In: Österreichische Namenforschung 13(1985) 39-73.

PoHL, H.D.: Slovenske (in slovanske) izposojenke v nemškem jeziku Koroške. In: Slavistična revija 37 (1989) 253-262 (= 1989a).

PoHL, H.D.: Kleine Kärntner Mundartkunde (mit Wörterbuch). Klagenfurt, Verlag Heyn 1989 (= $1989 \mathrm{~b}$ ).

PoHL, H.D.: Slovenske (in slovanske) izposojenke v nemškem jeziku Koroške (nadaljevanje in zaključek). In: Slavistična revija 38 (1990) 101-104.

PoHL, H.D.: Verzeichnis der Ortsnamen (Siedlungs-, Gewässer- und Bergnamen) des gemischtsprachigen Gebietes von Kärnten. In: Österreichische Namenforschung 20 (1992) 1-88.

PoHL, H.D.: Zur Sprache der Freisinger Denkmäler. Beziehungen zwischen der Sprache der Freisinger Denkmäler und den Ortsnamen Oberkärntens und Osttirols alpenslawischer bzw. slowenischer Herkunft. In: Zbornik Brižinski spomeniki. Ljubljana 1996, 311-321 (= 1996a).

PoHL, H.D.: Die Osttiroler Ortsnamen slawischer Herkunft. In: Österreichische Namenforschung 24(1996) 39$64(=1996 \mathrm{~b})$.

PoHL, H.D.: 222. Österreich. 223. Deutsch-Slowenisch. In: Kontaktlinguistik. Ein internationales Handbuch zeitgenössischer Forschung, 2. Halbband. Berlin-New York 1997, 1797-1812.

PoHL, H.D.: Slawische (slowenische) Bergappellativa in der österreichischen Oronymik. In: Studia celtica et indogermanica, Festschrift für Wolfgang Meid (hg. v. P. Anreiter - E. Jerem), Budapest 1999, $331-342$.

POHL, H.D.: Kärnten - deutsche und slowenische Namen. Koroška - slovenska in nemška imena. Kommentiertes zweisprachiges Verzeichnis der Siedlungs-, Berg- und Gewässernamen. In: Österreichische Namenforschung 28 (2000) Heft 2-3, 148 S. Lizenzausgabe in der Reihe Studia Carinthiaca Bd. XIX, Klagenfurt, Hermagoras 2000.

PoHL, H.D.: Bergnamen aus dem Kärntner Anteil an den Karnischen Alpen. In: Kärntner Landesgeschichte und Archivwissenschaft, Festschrift für Alfred Ogris, Klagenfurt 2001, 51-63.

PoHL, H.D.: Siedlungsgeschichte und Überlieferung von Ortsnamen slowenischer Herkunft in Osttirol und Kärnten (mit Ausblicken aufs übrige Österreich). In: Ortsnamen und Siedlungsgeschichte (Akten des Symposiums in Wien vom 28.-30.9.2000, hg. v. P. Ernst - I. Hausner - E. Schuster - P. Wiesinger), Heidelberg 2002, 177-189 (=2002a).

PoHL, H.D.: Aus Kärntens sprachlicher Vielfalt. Beiträge zur Kärntner Mundart- und Namenkunde. Fidibus, Zeitschrift für Literatur und Literaturwissenschaft 29 (2001 [2002])/2 (= 2002b).

PoHL, H.D.: Die Sprache der Kärntner Küche / Jezik koroške kuhinje. Ein Lexikon mit Ausblicken auf die österreichische und internationale Küche (für alle, die gerne kochen und wissen wollen, woher viele Küchenausdrücke kommen und was sie bedeuten). Klagenfurt, Hermagoras 2004.

PoPOVIC, I.: Geschichte der serbokroatischen Sprache. Wiesbaden 1960. 
RAMovš, F.: Kratka zgodovina slovenskega jezika I. Ljubljana 1936 (Nachdruck 1995).

RHAMm, K.: Talken und Geislitz (russisch toloknó und kisélj), zwei alte slawische Hafergerichte. In: Carinthia I 99 (1909), S. 209-222.

SCHMELLER, J. A.: Bayerisches Wörterbuch. München 1996 (Nachdruck von 1872-77).

SHEVELOV, G.Y.: A Prehistory of Slavic. Heidelberg 1964.

ŠMILAUER, V.: Př́ručka slovanské toponomastiky - Handbuch der slawischen Toponomastik, Praha 1970.

SNoJ, M., Slovenski etimološki slovar. Ljubljana, Mladinska knjiga 1997 (Druga, pregledana in dopolnjena izdaja 2003).

STEINHAUSER, W.: Zur Herkunft, Bildungsweise und siedlungsgeschichtlichen Bedeutung der niederösterreichischen Flur- und Ortsnamen, in: Jahrbuch für Landeskunde 1932, 1-48.

STEINHAUSER, W.: Slawisches im Wienerischen. Wien 1978.

WBMÖ: Wörterbuch der bairischen Mundarten in Österreich. Wien 1963ff.

ZDovc, P.: Die Mundart des südöstlichen Jauntales in Kärnten. Wien 1972. 
(außer allgemein bekannte Abkürzungen und Sprachbezeichnungen, bei den nur die Silbe ...isch fehlt)

\begin{tabular}{ll} 
A & Akkusativ \\
Bez. & Bezirk \\
BN & Bergname \\
D & Dativ \\
dt., Dt. & deutsch, Deutsch \\
FN & Flurname \\
G & Genitiv \\
GB & Gerichtsbezirk \\
Gem. Gemeinde \\
GN & Gewässername \\
HN & Hof- bzw. Hausname \\
I & Instrumental \\
idg. & indogermanisch \\
K & Kärnten \\
L & Lokativ \\
lat. & lateinisch \\
ma. & mundartlich \\
mhd. & mittelhochdeutsch \\
NÖ & Niederösterreich \\
ON & Ortsname \\
ÖNf & Österreichische \\
& Namenforschung \\
pl. & (Zeitschrift) \\
PN & Plural \\
rom. & Personenname \\
sg. & romanisch \\
skr. & Singular \\
slow. & serbisch und kroatisch (einschließlich bosnisch) \\
SN & slowenisch \\
St & Siedlungsname \\
urk. & Steiermark \\
& urkundlich \\
& \\
\hline
\end{tabular}




\section{Povzetek \\ SLAVIA SUBMERSA V AVSTRIJI: PREGLED IN POSKUS PREVREDNOTENJA}

Slovanska jezikovna oblika kot podlaga krajevnih imen na vzhodu in jugu Avstrije (Vzhodna Tirolska, Koroška, avstrijska Štajerska, Salzburg-Lungau, južni del Spodnje Avstrije ter vzhodni del Gornje Avstrije) ustreza "alpski slovanščini" oz. jeziku staroslovanskih "Brižinskih spomenikov". Nemške variante slovanskih imen je v glavnem mogoče razložiti kronološko, iz česar sledi, da je v Avstriji v visokem in poznem srednjem veku preko okvirov Koroške vladala močno razširjena slovansko-nemška jezikovna mešanica, kar se odraža tudi v starih izposojenkah. To jezikovno mešanico slovanstva v Avstriji zgodnjega srednjega veka so dovolj nazorno prikazali že drugi avtorji v zelo vsebinskih prispevkih. Ta jezik izkazuje nekatere posebnosti, ki jih v (današnjih) matičnih slovenskih področjih ni najti, med drugim tip krajevnega imena na -iče (glej 3.3) in pogost besedotvorni element -nik (najčešče -nig v nemškem prevodu, glej 3.2) kakor tudi nekaj besed (glej 4). Zastavlja se vprašanje, kako naj bi ta jezik poimenovali, "slovenščina" v današnjem smislu to ni, "alpska slovanščina" je preveč splošno, zato menim, da bi bilo najboljše ime karantanščina, ker je bila Slavia submersa avstrijskih alpskih dežel jezik (slovanskega) Karantanskega kraljestva, prve zgodovinske državne tvorbe prednikov današnjih Slovencev. V razdelkih 2-4 bo gradivo najprej predstavljeno in v 5 . razdelku ocenjeno. "Karantanščina" tvori jezikovno geografsko prehod od današnje slovenščine na jugu k zahodnoslovansko obarvani "erlaftalski slovanščini" (na Spodnjem Avstrijskem) na severu, največ skupnega ima z jezikom "Brižinskih spomenikov" in je predstavljala eno staroslovenskih narečij. 